\title{
Evaluación de la Vida Útil de una Pasta de Ajonjolí Azucarada mediante Pruebas Aceleradas
}

\author{
Katherine Paternina-Sierra, Diofanor Acevedo-Correa*, Piedad M. Montero-Castillo \\ Universidad de Cartagena, Facultad de Ingeniería, Grupo de Investigación en Innovación y Desarrollo \\ Agropecuario y Agroindustrial (IDAA), Avenida el Consulado, Calle 30 No. 48-152. Cartagena, \\ Bolívar-Colombia (e-mail: diofanor3000@gmail.com). \\ *autor a quien debe ser dirigida la correspondencia
}

Recibido Oct. 5, 2017; Aceptado Dic. 20, 2017; Versión final Feb. 26, 2018, Publicado Ago. 2018

\begin{abstract}
Resumen
El objetivo de esta investigación fue evaluar la vida útil de una pasta de ajonjolí azucarada con incorporación de plasma sanguíneo de bovino. Se realizaron pruebas aceleradas con ayuda del factor $Q_{10}$ y empleando el índice de peróxidos como indicador de deterioro. Para ello se evaluaron dos formulaciones con diferentes contenidos de pasta de ajonjolí ( $70 \%$ y $80 \%)$, almíbar (29\% y $19 \%$ ) y el mismo contenido en plasma ( $1 \%$ ). Ambas formulaciones se almacenaron a $15^{\circ} \mathrm{C}, 25^{\circ} \mathrm{C}$ y $35^{\circ} \mathrm{C}$ durante 80 días. Se realizaron tres muestreos para cada temperatura y los resultados obtenidos se utilizaron para definir la cinética de esta reacción de deterioro, la cual se asumió como de orden cero. El tratamiento elaborado con el $70 \%$ de pasta de ajonjolí presentó mejores características de vida útil con tiempos de 144 y 108 días de almacenamiento a $15^{\circ} \mathrm{C}$ y $25^{\circ} \mathrm{C}$, respectivamente.
\end{abstract}

Palabras clave: pasta de ajonjolí azucarada; índice de peróxidos; cinética; factor $Q_{10}$

\section{Evaluation of the Shelf Life of a Sweetened Sesame Paste by Accelerated Tests}

\begin{abstract}
The objective of this investigation was to evaluate the shelf-life of a sweetened sesame paste with addition of bovine blood plasma. Accelerated tests were performed employing the $Q_{10}$ factor and using peroxide index as an indicator of deterioration. For this, two formulations with different contents of sesame paste $(70 \%$ and $80 \%)$, syrup $(29 \%$ and $19 \%)$ and the same plasma content $(1 \%)$ were evaluated. Both formulations were stored at $15^{\circ} \mathrm{C}, 25^{\circ} \mathrm{C}$ and $35^{\circ} \mathrm{C}$ for 80 days. Three samples were taken for each temperature and the results obtained were used to define the kinetics of this deterioration reaction, which was assumed to be of zero order. The treatment elaborated with $70 \%$ of sesame paste presented better characteristics of shelf life with periods of 144 and 108 days of storage at $15^{\circ} \mathrm{C}$ and $25^{\circ} \mathrm{C}$, respectively.
\end{abstract}

Keywords: sweetened sesame paste; peroxide index; kinetics; $Q_{10}$ factor 


\section{INTRODUCCIÓN}

A pesar de los buenos datos que generan las pruebas de vida útil a tiempo real, estas requieren tiempos muy prolongados para la obtención del resultado final (Dak et al., 2014; Udomkun et al., 2016). Lo anterior, no es práctico para la empresa e investigadores, ya que todo este proceso se ve reflejado en mayores tiempos y gastos asociados (Ocheme et al., 2014; Lee et al., 2015). Por lo tanto, tener la información necesaria para determinar la vida útil de alimentos en un tiempo relativamente corto es de gran importancia (Min y Seok, 2014; Qi et al., 2014; Guzmán-Carrillo et al., 2016), por lo que los estudios de pruebas de almacenamiento o vida útil aceleradas se muestran como una solución rentable y eficaz en la determinación del tiempo de vida útil de un producto (Guzmán-Carrillo et al., 2016; Rao et al., 2012). Las pruebas de vida útil acelerados se refieren a cualquier método que sea capaz de evaluar la estabilidad del producto, basado en los datos que se obtienen en un en un período de tiempo significativamente más corto que el período de vida útil real del producto (Guzmán Carrillo et al., 2016; Derossi et al., 2016; Ramachandra y Rao, 2013). En este sentido, las cinéticas de orden cero, primer y segundo orden se han utilizado para estudiar las reacciones de degradación en productos alimenticios frescos y procesados, destacando las de orden cero, al ser las más usadas debido a su fácil manejo y buenos resultados la mayoría de las veces. En algunas investigaciones se opta por encontrar la velocidad de degradación ( $k$ ) a diferentes temperaturas de almacenamiento, prueba a la que se le llama de almacenamiento acelerado (Amodio et al., 2015). Usualmente se utiliza el modelo de Arrhenius para describir la dependencia de la temperatura a través de la energía de activación $\left(E_{a}\right)$ de la reacción (Sothornvit y Kiatchanapaibul, 2009).

El ajonjolí ofrece una excelente alternativa a la industria alimentaria, debido a su alto valor nutritivo en proteínas (17 a $23 \%$ ), en lípidos entre 42 a $50 \%$ en ácidos grasos monoinsaturados como el oleico y poliinsaturados como el linoléico, minerales como calcio, hierro y potasio, y a nivel de vitaminas se encuentran la E, K, B3, B5, B6, el ácido, fólico, biotina, inositol y colina (Julio et al., 2015; Montero et al., 2015). En este sentido, la pasta de ajonjolí (Sesamum indicum L.), también conocida como tahini, es definida como una dispersión coloidal compleja de aceite, rica en constituyentes como proteínas y lípidos (Çiftçi et al., 2008). Esta es obtenida mediante la molienda de las semillas descascarilladas y tostadas, sin la adición o eliminación de ningún componente nativo (Aloui et al., 2016). Esta pasta contiene una buena cantidad de calcio, hierro, potasio, fósforo y vitaminas B, C y E (Prasad et al., 2012). En cuanto a la pasta de sésamo dulce (halawa tehineh, también conocido como halaweh, halva, helva y como "Turrón" en griego), esta es un producto alimenticio dulce común en Grecia y Oriente Medio que consiste en una mezcla de azúcar tratada térmicamente, aceites vegetales (principalmente aceite de palma) y tahine. Pueden añadirse algunos sabores naturales, como vainilla y frutos secos (Abu-Jdayil, 2004; Borchani et al., 2010; Goulas et al., 2007; Elleuch et al., 2011). Un líquido extraído de la raíz de la saponaria se utiliza como aditivo en la preparación de halawa tehineh, el cual tiene un efecto potencial sobre el color y la consistencia de esta pasta dulce y actúa como un emulsionante. El halawa tehineh consiste principalmente en $50 \%$ en peso de pasta de sésamo, $25 \pm 35 \%$ peso de sacarosa y $12 \pm 25 \%$ de glucosa (Abu-Jdayil, 2004). Asímismo, las proteínas del plasma sanguíneo poseen características favorables para su uso en la industria de los alimentos, destacando su alto valor nutricional, sus propiedades como agente emulsificante, espumante, ligante y gelificante. Investigaciones realizadas en la formulación de alimentos con plasma sanguíneo, reportan que la adición de éste mejora el rendimiento, aumenta el contenido proteico y reduce el contenido graso de los productos, sin que ello genere un efecto negativo en la aceptabilidad (Montero et al., 2015; Julio et al., (2015), Tirado et al., 2015).

Teniendo en cuenta que uno de los principales factores que reducen el tiempo de vida útil de los productos oleaginosos son los abusos de temperatura durante el proceso de almacenamiento, el objetivo de esta investigación fue determinar la vida útil de la pasta de ajonjolí azucarada mediante pruebas aceleradas, empleando el índice de peróxidos expresado en miliequivalentes de oxígeno activo por kg de grasa, como indicador de una cinética de deterioro de orden cero a tres diferentes temperaturas $\left(15^{\circ} \mathrm{C}, 25^{\circ} \mathrm{C}\right.$ y $\left.35^{\circ} \mathrm{C}\right)$.

\section{MATERIALES Y MÉTODOS}

Se describe la formulación de los mejores tratamientos de la pasta de ajonjolí azucarada obtenidos en estudios preliminares. Luego se describe el proceso de evaluación de las características microbiológicas y finalmente se detalla el estudio de vida útil mediante el índice de peróxidos del producto a condiciones aceleradas con ayuda del factor $\mathrm{Q}_{10}$

\section{Resultados preliminares}

Las pastas de ajonjolí azucaradas que mejores características obtuvieron en estudios preliminares en cuanto a estabilidad, calidad sensorial y características bromatológicas fueron seleccionadas para evaluar sus características microbiológicas y su vida útil. En este orden, se emplearon dos tratamientos, T1 con $70 \%$ de 
pasta de ajonjolí, $29 \%$ almibar y $1 \%$ plasma sanguineo, y T2 con $80 \%$ pasta de ajonjolí, $19 \%$ almibar y $1 \%$ plasma sanguineo.

\section{Características microbiológicas}

El análisis microbiológico al producto se realizó según la Norma Técnica Colombiana (NTC) 1055 de 2007 para productos de molinería y pastas alimenticias (NTC, 2007). Se realizó el recuento total de aerobios mesófilos, coliformes totales y fecales y recuento de hongos y levaduras (UFC/g) mediante recuento en placa.

\section{Estudio de vida útil}

La determinación de vida útil de los productos finales se realizó teniendo en cuenta el aumento del índice de peróxidos del producto a condiciones aceleradas con ayuda del factor $\mathrm{Q}_{10}$.

\section{Índice de peróxidos (IP)}

El índice de peróxidos (IP) es la cantidad (expresada en meq de $\mathrm{O}_{2} / \mathrm{kg}$ de grasa) de peróxidos en la muestra que ocasionan la oxidación del yoduro potásico en las condiciones de trabajo descritas (García y Molina, 2008; Guzmán-Carrillo et al., 2016). La muestra problema, disuelta en ácido acético y cloroformo, se trató con solución de yoduro potásico. El yodo liberado se valoró con solución valorada de tiosulfato sódico según el método Standard Internacional ISO 3960 de la técnica de determinación de IP bajo los lineamientos de la A.O.A.C. 965.33 (A.O.A.C., 2012). Los ensayos se realizaron por triplicado. Finalmente el IP se calculó mediante la ecuación (1).

$$
\mathrm{IP}=\frac{1000\left(\mathrm{~V}-\mathrm{V}_{0}\right) \mathrm{C}}{\mathrm{m}}
$$

Donde IP (meq de $\mathrm{O}_{2} / \mathrm{kg}$ ) es el índice de peróxidos en aceite; $\mathrm{V}\left(\mathrm{cm}^{3}\right)$ es el volumen de solución de tiosulfato de sodio utilizado para la determinación, $V_{0}\left(\mathrm{~cm}^{3}\right)$ es el volumen de solución de tiosulfato de sodio utilizado en el blanco, C (mol/L) es la concentración de la solución de tiosulfato y $\mathrm{m}(\mathrm{g})$ es la masa de la muestra.

\section{Periodicidad de análisis}

Autores indican que la cantidad mínima de temperaturas para conducir un estudio de vida útil mediante pruebas aceleradas son tres. Para este estudio se establecieron las temperaturas de $15^{\circ} \mathrm{C}, 25^{\circ} \mathrm{C}$ y $35^{\circ} \mathrm{C}$. Las temperaturas se escogieron para establecer una diferencia de $10{ }^{\circ} \mathrm{C}$ y poder calcular el valor de $\mathrm{Q}_{10}$ que representa la razón de las constantes de velocidad de reacción a las temperaturas mencionadas (García y Molina, 2008). Las muestras fueron analizadas los días $0,5,10,15,20,25,35,50,65$ y 80.

\section{Modelo para la degradación cinética}

Por su simplicidad y buenos resultados en estudios anteriores (Amodio et al., 2015; Figueroa et al., 2012; García et al., 2011; García y Molina, 2008), en esta investigación se trabajó con un modelo de orden cero como el de la ecuación (2). Integrando la ecuación (2) y reacomodando, se tiene la ecuación (3), la cual representa una línea recta con pendiente k; siendo k la constante específica de reacción y cuyo valor depende de la temperatura.

$$
\begin{aligned}
& -\frac{\partial X}{\partial t}=k \\
& X_{f}=X_{0}-k t
\end{aligned}
$$

Puesto que la constante de velocidad de reacción está en función de la temperatura, esta dependencia es descrita por la ecuación de Arrhenius, según la ecuación (4).

$$
\mathrm{k}=\mathrm{A} e^{\left(\frac{-E_{a}}{R T}\right)}
$$

Al aplicar logaritmos a ambos lados de la ecuación (4) se obtiene la ecuación de una línea recta con pendiente $E_{a} / R$, tal como se expresa en la ecuación (5). El término $E_{a}$ puede evaluarse para conocer el valor de la energía de activación, donde $k$ es constante de velocidad de reacción, $A$ es el factor de frecuencia, $R$ es la constante de los gases ideales $\left(8,31 \mathrm{~J} / \mathrm{mol}^{*} \mathrm{~K}\right)$ y $\mathrm{T}$ es la temperatura absoluta $(\mathrm{K})$. 
$\operatorname{Ln} k=\frac{E_{a}}{R} \cdot \frac{1}{T}+\operatorname{Ln} A$

\section{Cálculo del parámetro $Q_{10}$}

El parámetro $Q_{10}$ se define como la razón entre la constante de velocidad a una temperatura $(T)$ y la constante de velocidad a otra temperatura $\left(T+10^{\circ} \mathrm{C}\right)$. Este no es constante sino que depende de $\mathrm{E}_{\mathrm{A}}$ y la temperatura absoluta $T$. El parámetro $Q_{10}$ se calcula de acuerdo a la ecuación (6). Donde $\mathrm{k}_{\mathrm{T}}$ es la constante de reacción a $\mathrm{T}_{1}$ y $\mathrm{k}_{\mathrm{T}+10}$ es la constante $\mathrm{a} \mathrm{T}_{2}=\mathrm{T}_{1}+10^{\circ} \mathrm{C}$. $\mathrm{VU}_{\mathrm{T}}$ es la vida útil del producto a $\mathrm{T}_{1}$ y $\mathrm{VU}_{\mathrm{T}+10}$ es la vida de anaquel a $T_{2}$.

$$
\mathrm{Q}_{10}=\frac{\mathrm{k}_{\mathrm{T}+10}}{\mathrm{k}_{\mathrm{T}}}=\mathrm{e}^{\left(\frac{10 \mathrm{E}_{\mathrm{a}}}{\mathrm{RT}(\mathrm{T}+10)}\right)}=\frac{\mathrm{VU}_{\mathrm{T}}}{\mathrm{VU}_{\mathrm{T}+10}}
$$

\section{RESULTADOS Y ANÁLISIS}

En la Tabla 1 se muestra la evaluación microbiológica de la pasta de ajonjolí azucarada con incorporación de plasma sanguíneo. Con estos resultados se demuestra que la pasta es un producto que puede ser utilizado en la industria alimentaria, sin que ponga en riesgo la salud del consumidor, ya que cumple con todos los parámetros evaluados requeridos por la NTC 1055 (NTC, 2007). Cabe señalar que el crecimiento reducido de patógenos en pastas de ajonjolí podría atribuirse a la baja actividad de agua (aproximadamente 0,31 ) y alto contenido de grasa $(56,8 \%)$, lo que hace a este tipo de productos un entorno de crecimiento desfavorable (Al-Nabulsi et al., 2013). Estos resultados son similares a los encontrados por Garrido et al. (2010) en su estudio con pasta untable de pavo enriquecida con fibra, vitaminas y minerales antioxidantes como ingredientes funcionales para el adulto mayor.

Tabla 1: Análisis microbiológicos de la pasta azucarada de ajonjolí.

\begin{tabular}{|l|c|c|}
\hline \multicolumn{1}{|c|}{ Parámetro } & Resultado & Límite según NTC 1055 (NTC, 2007) \\
\hline Recuento de aerobios mesófilos (UFC/g) & 1880 & 2000 \\
\hline Recuento de mohos (UFC/g) & 9 & 4000 \\
\hline Recuento de levaduras (UFC/g) & 8 & 4000 \\
\hline Recuento de coliformes totales (UFC/g) & 200 & $<10$ \\
\hline Recuento de coliformes fecales (UFC/g) & 2,6 & $<10$ \\
\hline Recuentos S. aureus Coagulasa positiva (UFC/g) & 90 & 100 \\
\hline $\begin{array}{l}\text { Recuento de esporas de Clostridium sulfito-reductor } \\
\text { (UFC/g) }\end{array}$ & 9 & $<10$ \\
\hline Detección de Salmonella spp. & Ausente & Ausente \\
\hline
\end{tabular}

La contaminación de este tipo de pastas pueden estar asociadas con malas prácticas de saneamiento, diseño de instalaciones y equipamiento deficiente, mantenimiento inadecuado, malas prácticas operacionales o de fabricación, control inadecuado de ingredientes y otros factores (Podolak et al., 2010). Asimismo, Al-Nabulsi et al, 2013, reportaron que el tahini o sus productos pueden estar contaminados con varios tipos de patógenos transmitidos por los alimentos durante el procesamiento y la preparación, por lo que la prevención de la contaminación previa o posterior al procesamiento, junto con la adopción de prácticas higiénicas adecuadas, son esenciales para minimizar el riesgo asociado con los patógenos transmitidos en estos alimentos.

Por su parte, Ayaz et al. (1986), en su investigación sobre la calidad microbiana de tahini fabricado en Arabia Saudita, encontraron que los rangos de bacterias aerobias, coliformes, $S$. aureus, Bacillus cereus $y$ Clostridium perfringens fueron de 1,3 $\log _{10} \mathrm{CFU} / \mathrm{g}-5,2 \log _{10} \mathrm{CFU} / \mathrm{g},<1,0 \log _{10} \mathrm{CFU} / \mathrm{g}-2,5 \log _{10} \mathrm{CFU} / \mathrm{g},<1,0$ $\log _{10} \mathrm{CFU} / \mathrm{g}-2,6 \log _{10} \mathrm{CFU} / \mathrm{g},<1,0 \log _{10} \mathrm{CFU} / \mathrm{g}-2,4 \log _{10} \mathrm{CFU} / \mathrm{g}$ y $1,0 \log _{10} \mathrm{CFU} / \mathrm{g}-2,0 \log _{10} \mathrm{CFU} / \mathrm{g}$, respectivamente, en tahini de muestras recogidas en 10 plantas en Arabia Saudita. Además, Karam (2010) en su estudio sobre la determinación y validación de criterios microbianos para tahini y halawi, encontraron E. coli en el $17 \%$ de las muestras.

\section{Estudio de vida útil}

Los resultados del IP de los tratamientos T1 y T2 en función del tiempo para cada temperatura se muestran en las Figuras 1 y 2 , respectivamente. Se puede observar que el IP de las pastas de ajonjolí aumentó respecto al tiempo y a mayores temperaturas. Las regresiones lineales obtenidas de esta figura se presentan en las 
ecuaciones (7), (8), (9), (10), (11) y (12). La tendencia muestra que los incrementos del IP ocurren a una velocidad de deterioro mayor a medida que aumenta la temperatura de almacenamiento, lo cual implica un aumento de la rancidez del producto debida a las reacciones químicas que ocurren en los productos oleaginosos como la pasta de ajonjolí. Es así como $\mathrm{T} 1$, a $35^{\circ} \mathrm{C}$ presentó un IP de $1,10 \mathrm{meqO}_{2}$ a los 20 días de almacenamiento; mientras que a $15^{\circ} \mathrm{C}$ se determinó que para el día 65 fue de solo 2,11 meqO${ }_{2}$.

Esta tendencia se presentó de igual forma para $\mathrm{T} 2\left(1,55 \mathrm{meqO}_{2}\right.$ a los 20 días y $35^{\circ} \mathrm{C}$; y $2,25 \mathrm{meqO}_{2}$ a los 50 días y $15^{\circ} \mathrm{C}$ ). Estos resultados coinciden con los de Rondón et al. (2004), quienes reportaron en su estudio sobre la vida útil de un análogo comercial de mayonesa, aumentos significativos en el IP a medida que aumentaba el tiempo y la temperatura, con un valor de 2,0 meqO $_{2}$ presentado a los 21 días de almacenamiento a $45^{\circ} \mathrm{C}$ y solo hasta el día 60 a $35^{\circ} \mathrm{C}$ se determinó el límite de rancidez $\left(2,09 \mathrm{meqO}_{2}\right)$. Así mismo, estudios por García \& Molina (2008) coinciden con los resultados obtenidos en el presente estudio.

$$
\begin{aligned}
& I P=0,0393 t-0,3086 \\
& I P=0,0465 t-0,2801 \\
& I P=0,0708 t-0,3967 \\
& I P=0,0526 t-0,2596 \\
& I P=0,0691 t-0,12277 \\
& I P=0,0856 t-0,1567
\end{aligned}
$$

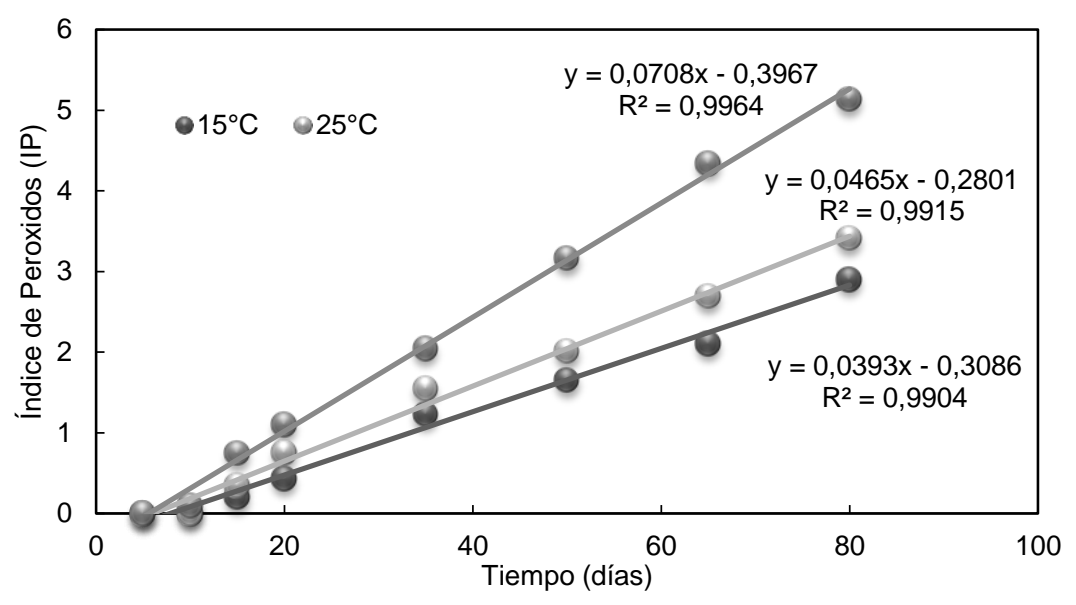

Fig. 1: Índice de peróxidos de T1 en función del tiempo.

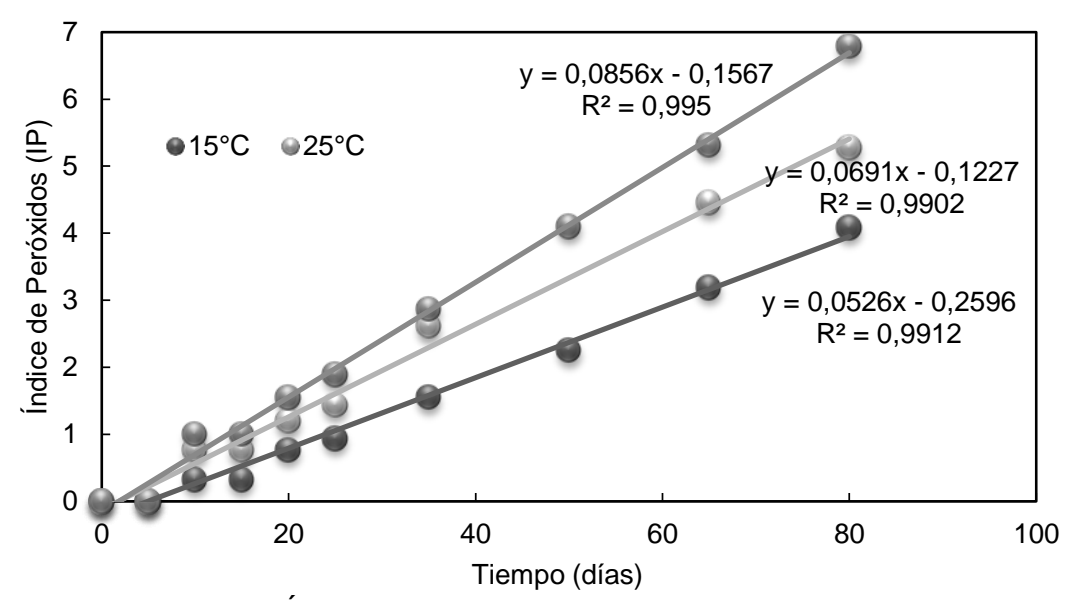

Fig. 2: Índice de peróxidos de T2 en función del tiempo. 
El desarrollo de peróxidos se atribuye al deterioro oxidativo de la fracción grasa durante el almacenamiento, proceso que transcurrirá aun cuando existan aditivos que lo retrasen (Yang et al., 2013), generando cambios en el aroma y sabor, y por ende disminución de la vida útil y aceptación de éstos. La rancidez oxidativa resulta de la formación de hidroperóxidos en la etapa de iniciación, etapa que se desarrolla de forma lenta, estos se acumulan durante la etapa de propagación, para degradarse finalmente y con mayor velocidad en la etapa de terminación, formando aldehídos y cetonas responsables del olor rancio (Rondón et al., 2004).

Con las constantes obtenidas, representadas por las pendientes de las ecuaciones (7), (8), (9), (10), (11) y (12) para las tres temperaturas estudiadas, se aplicó el modelo de Arrhenius, en la forma como se expresa en la ecuación (5). De la regresión lineal que se muestra en las Figuras 3 y 4 se obtienen las ecuaciones (13) y (14) respectivamente, necesarias para el cálculo de la energía de activación.

$$
\begin{aligned}
& \operatorname{Ln} \mathrm{k}=5,7422-2599,9 \frac{1}{T} \\
& \operatorname{Ln} \mathrm{k}=4,5721-2164,1 \frac{1}{T}
\end{aligned}
$$

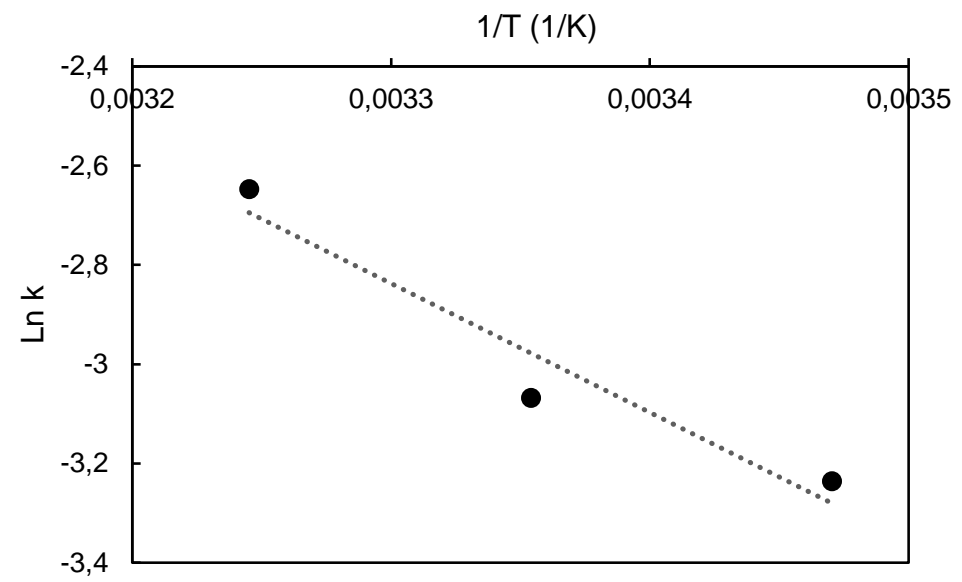

Fig. 3: Ln k en función de 1/T de T1

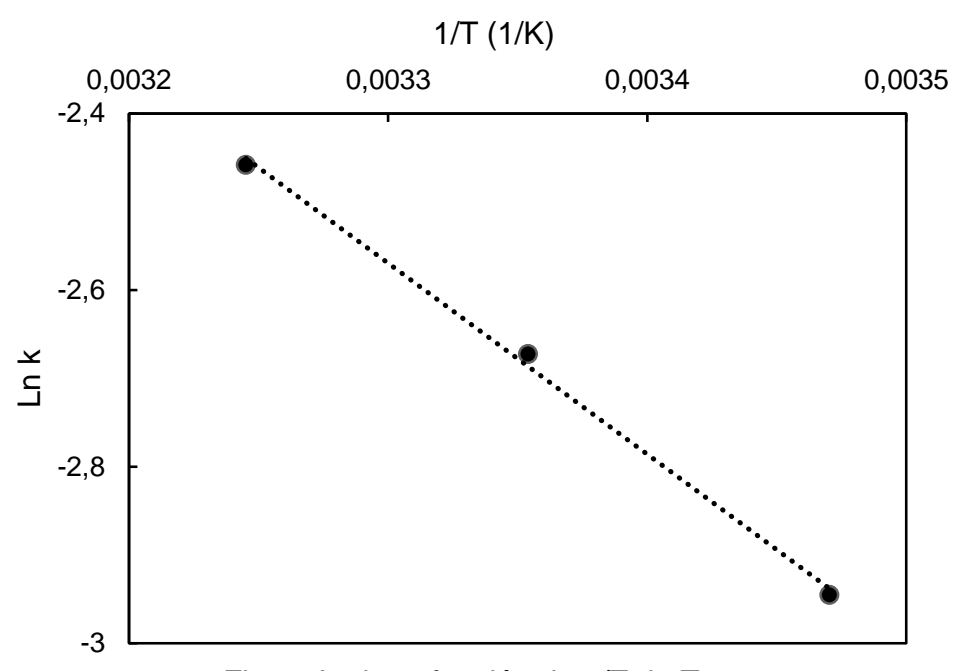

Fig. 4: Ln k en función de 1/T de T2

Teniendo en cuenta la estructura de la ecuación (5), la energía de activación para T1 y T2 fue de 21.616,79 $\mathrm{J} / \mathrm{mol}$ y $17.993,34 \mathrm{~J} / \mathrm{mol}$, respectivamente. Estos resultados coinciden con lo que afirman Arroyo et al. (2016), pues según su estudio sobre las propiedades reológicas de pulpa edulcorada de tomate de árbol (Cyphomandrabetacea), la energía de activación ( $\left.E_{a}\right)$ resulta más elevada cuando aumenta la concentración de sacarosa, lo que demuestra un mayor efecto de la temperatura sobre la viscosidad. Por otra parte, Torres et al. (2001) en su estudio sobre la estimación de la vida útil de una fórmula dietética en función de la disminución de lisina disponible, indicaron que la energía de activación para reacciones de oxidación de lípidos se encuentra en el ámbito de $41.842 \mathrm{~J} / \mathrm{mol}$ a $104.605 \mathrm{~J} / \mathrm{mol}$. 
En este sentido, la energía de activación de los tratamientos de este estudio no están dentro del intervalo para una reacción de oxidación de lípidos, lo cual se traduce en una vida útil prolongada para el producto; sin embargo, no se descarta la posibilidad de que otras reacciones de deterioro estén sucediendo al mismo tiempo (Yang et al., 2013). Cabe señalar que otros autores han utilizado este tipo de modelo por su eficacia y buenos resultados (García y Molina, 2008; García et al., 2011; Figueroa et al., 2012; Amodio et al., 2015).

\section{Cálculo del parámetro $Q_{10}$ para el tratamiento $T 1$ y $T 2$}

Remplazando los valores de $E_{a}, R, T$ y $(T+10)$ en la ecuación (6) y despejando $Q_{10}$ se encontró un valor de 1,35 y 1,28 para T1 y T2 respectivamente, este valor indica que la velocidad de la reacción de deterioro se acelera 1,35 y 1,28 veces por cada $10 \stackrel{\circ}{ } \mathrm{C}$ de aumento en la temperatura, o lo que es igual, la vida útil de este producto decrece 1,35 y 1,28 veces por cada $10 \stackrel{\circ}{C}$ de aumento en la temperatura para los tratamientos respectivamente. Según las evaluaciones de paneles sensoriales efectuadas con jueces entrenados, se obtuvo un valor de índice de peróxidos de $5,14 \mathrm{meqO}_{2} / \mathrm{kg}$ y $6,79 \mathrm{meqO}_{2} / \mathrm{kg}$ para $\mathrm{T} 1$ y T2 respectivamente para los cuales dichos jueces consideraron que el producto ya no reunía las cualidades necesarias para el consumo. Con estos valores y las ecuaciones (7), (8), (9), (10), (11) y (12) se estimaron los valores puntuales de vida útil de la pasta de ajonjolí azucarada, para las temperaturas de almacenamiento $15{ }^{\circ} \mathrm{C}, 25{ }^{\circ} \mathrm{C}$ y $35^{\circ} \mathrm{C}$.

Se graficó el logaritmo de la vida útil a $15{ }^{\circ} \mathrm{C}, 25{ }^{\circ} \mathrm{C}$ y $35^{\circ} \mathrm{C}$. La pseudo-transformación resultante para el tratamiento T1 se puede observar en la Figura 5, y para T2 en la Figura 6. Esta pseudo-transformación se puede utilizar siempre y cuando los ámbitos de temperatura sean estrechos (García y Molina, 2008). Mediante regresión lineal obtenida de la Figura 5, resultó la ecuación (15) con la que se estimó la vida útil de la pasta de ajonjolí azucarada para el tratamiento T1 a diferentes temperaturas de almacenamiento, donde T está en ㄷ. De igual forma, de la Figura 6 se obtuvo la ecuación (16), para estimar la vida útil del tratamiento T2.

Log vida útil T1= 2,3447-0,0124T

Log vida útil T2=2,2827-0,0109T

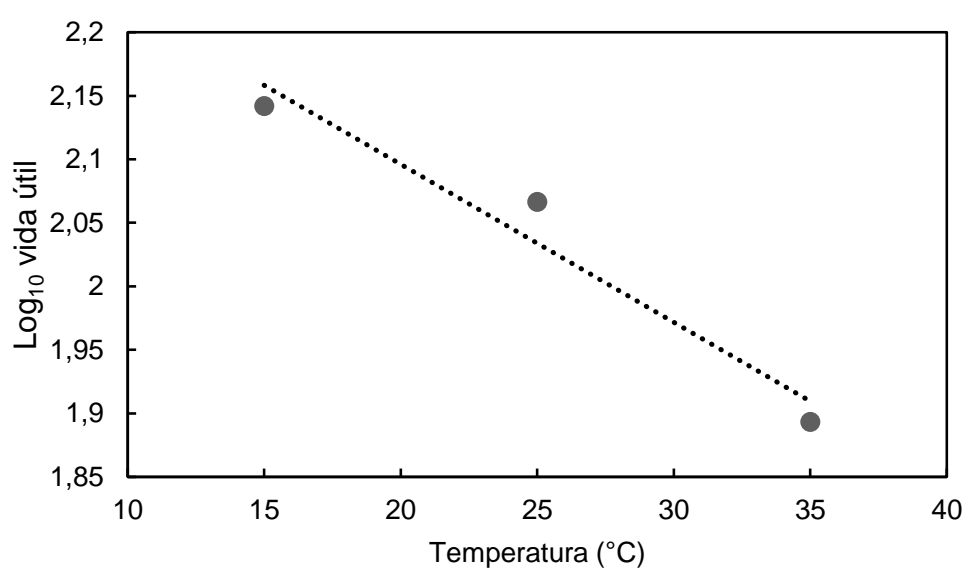

Fig. 5: $\log _{10}$ de vida útil en función de la temperaturas para T1

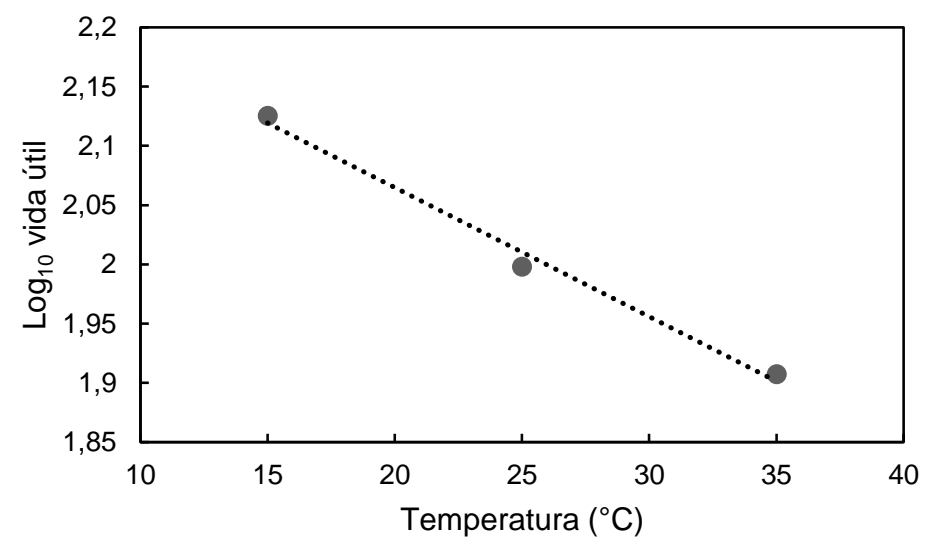

Fig. 6: $\log _{10}$ de vida útil en función de la temperaturas para T2 
Las ecuaciones (15) y (16) se despejaron y se obtuvo la vida útil de T1 y T2, tal como se observa en la Tabla 2.

Tabla 2: Valores de vida útil de T1 y T2.

\begin{tabular}{|c|c|c|}
\hline Temperatura $\left({ }^{\circ} \mathrm{C}\right)$ & Vida útil de T1 (días) & Vida útil de T2 (días) \\
\hline 15 & 144 & 131 \\
\hline 25 & 108 & 102 \\
\hline 35 & 81 & 79 \\
\hline
\end{tabular}

Los valores de vida útil encontrados por este método son una confirmación de los hallados mediante el cálculo del Índice de peróxidos. La diferencia entre T1 y T2 en cuanto al tiempo de vida útil presentados en la Tabla 2 puede ser debido al porcentaje de ajonjolí de estos en las formulaciones, pues al tener T2 un $10 \%$ más de pasta de ajonjolí que T1 y por ende un mayor contenido de grasa, por ser la semilla rica en aceite, esto puede ayudar al incremento del IP y por tal motivo una ligera menor vida útil frente al tratamiento T1, cabe señalar que esta situación se presenta principalmente a temperaturas menores a $25^{\circ} \mathrm{C}$, las cuales ofrecen mejores tiempos de conservación del producto. Sin embargo, a medida que aumenta la temperatura, se evidencian semejanzas en el tiempo de vida útil de ambos tratamientos.

\section{CONCLUSIONES}

De acuerdo al estudio de vida útil mediante pruebas aceleradas, se observó un aumento del índice de peróxidos de la pasta de ajonjolí azucarada con el trascurso de los días y a mayores temperaturas de almacenamiento, logrando establecerse que el tratamiento con $70 \%$ de pasta de ajonjolí, $29 \%$ de almíbar y $1,00 \%$ de plasma (T1) presentó mejores características de vida útil con tiempos de hasta 144 días a $15^{\circ} \mathrm{C}$, por lo cual resulta óptimo para usarse en el desarrollo de productos de pastelería. Por su parte, los análisis microbiológicos realizados indicaron que la pasta de ajonjolí azucarada fue elaborada bajo condiciones de aseo y desinfección, cumpliendo con lo establecido en la norma colombiana.

\section{AGRADECIMIENTOS}

Los autores agradecen al Departamento Administrativo de Ciencia, Tecnología e Innovación (COLCIENCIAS) por la financiación del proyecto 110766441920 a través de Convocatoria 664.

\section{REFERENCIAS}

AOAC., Association of Official Analytical Chemists, Official Methods of Analysis, 19th Ed. AOAC, Arlington, VA, USA (2012)

Abu-Jdayil, B., Flow properties of sweetened sesame paste (halawa tehineh), European Food Research and Technology, 219(3), $265-272$ (2004)

Abu-Jdayil, B., K. Al-Malah y H. Asoud, Rheological characterization of milled sesame (tehineh), Food Hydrocoll., 16, 55 $61(2002)$

Aimaretti, N. y otros cuatro autores, Desarrollo de una pasta untable vegetal a base de harina de grano entero de sorgo y de mijo, Revista Española de Nutrición Humana y Dietética, 17(2), 54 - 60 (2013)

Al-Nabulsi, A. y otros seis autores, Survival of E. coli O157: H7 and Listeria innocua in tahini (sesame paste), Journal of Food, Agriculture \& Environment, 11 (3-4), 303-306 (2013)

Aloui, F., y otros tres autores, Optimization of oil retention in sesame based halva using emulsifiers and fibers: an industrial assay, J. Food Sci. Technol., 53, 1540-1550 (2016)

Amodio, M.L. y otros tres autores, A study of the estimated shelf life of fresh rocket using a non-linear model, J. Food Eng., 150, 19-28 (2015)

Arroyo, E., K. Contreras y J. Figueroa, Propiedades reológicas de pulpa edulcorada de tomate de árbol (Cyphomandrabetacea), Agronomía Colombiana, 34 (1Supl.), S 419-S421 (2016)

Ayaz, M., W.N. Sawaya y A. Al-Sogair, Microbial quality of Tehineh manufactured in Saudi Arabia, J. Food Prot., 49, 504506 (1986)

Borchani, C., S. Besbes, C. Blecker y H. Attia, Chemical characteristics and oxidative stability of sesame seed, sesame paste, and olive oils, Journal of Agricultural Science and Technology, 12, 585-596 (2010)

Çiftçi, D. y otros tres autores, Colloidal stability and rheological properties of sesame paste, J. Food Eng., 87, 428-435 (2008)

Dak, M., V.R. Sagar y S.K. Jha, Shelf-life and kinetics of quality change of dried pomegranate arils in flexible packaging, Food Packag., Shelf Life, 2, 1-6 (2014) 
Derossi, A. y otros cuatro autores, Application of multivariate accelerated test for the shelf life estimation of fresh-cut lettuce, Journal of Food Engineering, 169, 122-130 (2016)

Elleuch, M., D. Bedigian, A. Zitoun y N. Zouari, Sesame (Sesamum indicum L.) seeds in food, nutrition and health. Nuts \& seeds in health and disease prevention, Elsevier Inc., 1029-1036 (2011)

Figueroa, O.A., J.E. Zapata y G.A. Gutierrez, Modelamiento de la cinética de hidrólisis enzimática de proteínas del plasma bovino, Rev. EIA, 17, 71-84 (2012)

García, C., G. Chacón y M.E. Molina, Evaluación de la vida útil de una pasta de tomate mediante pruebas aceleradas por temperatura, Ingeniería, 21, 31-38 (2011)

García, C. y M.E. Molina, Estimación de la vida útil de una mayonesa mediante pruebas aceleradas, Ingeniería, 18, 57-64 (2008)

Goulas, A. E., P. Zygoura, A. Karatapanis, D. Georgantelis, y M. G. Kontominas, Migration of di (2-ethylhexyl) adipate and acetyltributyl citrate plasticizers from foodgrade PVC film into sweetened sesame paste (halawa tehineh): Kinetic and penetration study, Food and chemical toxicology, 45(4), 585-591 (2007)

Guzmán, L.E. y otros cuatro autores, Estudio de la vida útil de jamón de cerdo mediante pruebas aceleradas, Interciencia, 41(7), 488-491 (2016)

ICONTEC, Norma Técnica Colombiana para Productos de molinería, Pastas alimenticias, Bogotá D.C. (2007)

Julio, L.C., P. M. Montero y D. Acevedo, Calidad y aceptabilidad de chorizos formulados con plasma sanguíneo bovino y pasta de ajonjolí, Información Tecnológica, 26(3), 25-32 (2015)

Karam, L.A., The Determination and Validation of Microbial Criteria for Tahini and Halawi Products; Tesis de maestría, Dpto. Nutric. y Ciencia de los Alimentos, American University of Beirut, Lebanon, 150 p. (2010)

Lee, E.S. y otros cinco autores, Quality evaluation and estimation of shelf life of retort-pouched tomato-based and Korean traditional fermented food-based sauces, J. Kor. Soc. Appl. Biol. Chem., 5, 229-236 (2015)

Min, M. y Y. Seok, Temperature management for the qualit y assurance of a perishable food supply chain, Food Contr., 40, 198-207 (2014)

Montero, P.M., D. Acevedo, A.J. Arnedo y N. K. Miranda, Efecto de la Incorporación de Plasma Sanguíneo y Pasta de Ajonjolí en la Fabricación de un Embutido tipo Salchicha, Información Tecnológica, 26(6), 55-64 (2015)

Ocheme, B. y otros tres autores, Prediction of Shelf Life of Dakuwa (Nigerian cereal/groundnut snack) Using $\mathrm{pH}$ as Index of Acceptability, Food Sci. Qual. Manag., 24, 16-23 (2014)

Podolak, R., y otros cuatro autores, Sources and risk factors for contamination, survival, persistence, and heat resistance of Salmonella in low moisture foods, J. Food Prot., 73, 1919-1936 (2010)

Prasad, N., K.R. Sanjay y D. Prasad, A Review on Nutritional and Nutraceutical Properties of Sesame, J. Nutr. Food Sci., 2, 1-6 (2012)

Qi, L., y otros cuatro autores, C2SLDS: A WSN-based perishable food shelf-life prediction and LSFO strategy decision support system in cold chain logistics, Food Control, 38, 19-29 (2014)

Ramachandra, C.T. y P.S. Rao, Shelf-life and colour change kinetics of Aloe vera gel powder under accelerated storage in three different packaging materials, J. Food Sci. Technol., 50, 747-754 (2013)

Rao, Q. y otros tres autores, Accelerated shelf-life testing of quality loss for a commercial hydrolysed hen egg white powder, Food Chem., 135, 464-472 (2012)

Reshma, M.V. y otros seis autores, Extraction, separation and characterisation of sesame oil lignan for nutraceutical applications, Food Chem., 120, 1041-1046 (2010)

Rondón, E. y F. Ortega, Estimación de la vida útil de un análogo comercial de mayonesa utilizando el factor de aceleración Q10, Revista de la Facultad de Agronomía, 21(1), 68-83 (2004)

Sothornvit, R. y P. Kiatchanapaibul, Quality and shelf life of washed fresh-cut asparagus in modified atmosphere packaging, LWT - Food Sci. Technol., 42, 1484-1490 (2009)

Tirado, D.F., P.M. Montero y D. Acevedo, Sensory acceptability and microbiological quality of rice based drinks and bovine and porcine plasma, Inf. Tecnológica, 26, 45-54 (2015)

Torres, A., M. Guerra e Y. Rosquete, Estimación de la vida útil de una fórmula dietética en función de la disminución de lisina disponible, Food Science and Technology, 21(2), 129-133 (2001)

Udomkun, P. y otros cinco autores, Compositional and functional dynamics of dried papaya as affected by storage time and packaging material, Food Chem., 196, 712-719 (2016)

Yang, N. y otros cinco autores, Impact of flavour solvent (propylene glycol or triacetin) on vanillin, 5-(hydroxymethyl)furfural, 2,4-decadienal, 2,4-heptadienal, structural parameters and sensory perception of shortcake biscuits over accelerated shelf life testing, Food Chem., 141, 1354-1360 (2013) 
